\title{
Prescription of eye drops
}

\author{
E P O’Sullivan, R Malhotra, C Migdal
}

\begin{abstract}
The aim of this study was to assess whether patients have their ocular drops correctly prescribed during nonophthalmic admissions to hospital. A retrospective review of notes of patients who were admitted to hospital for general medical or surgical care, while on regular eye drops at the time of admission was performed. Twenty two patients were on regular ocular medication when admitted. Only seven out of 22 patients had their eye drops correctly prescribed. Furthermore, six patients had been prescribed topical $\beta$-blockers, yet suffered from medical conditions that may have been aggravated by these drops. These findings demonstrate that the majority of patients on drops do not have their medication correctly prescribed during non-ophthalmic admissions to hospital. Also topical $\beta$-blockers continue to be inappropriately prescribed.
\end{abstract}

(Postgrad Med f 2001;77:654-655)

Keywords: eye drops; non-ophthalmic admissions

Medications prescribed in the form of eye drops are important in treating ocular conditions (for example glaucoma or uveitis) but they may also have adverse systemic effects. ${ }^{1}$ It has been demonstrated that patients admitted to hospital for non-ophthalmic reasons do not always receive their eye drops as prescribed. ${ }^{2}$ The aim of this study was to assess the current level of incorrect or even inappropriate prescribing of eye drops.

\section{Methods}

This retrospective review was carried out at the Western Eye Hospital which is part of St Mary's NHS Trust but is essentially a standalone specialist eye unit situated some distance from the general medical and surgical wards, which are based at St Mary's Hospital. Ophthalmic notes are kept on site at the Western Eye Hospital. A list of all patients, who had been admitted to the medical or surgical wards at St Mary's Hospital for more than two days between January 1996 and December 1998, and who also had Western Eye Hospital ophthalmic notes, was provided by the $\mathrm{St}$ Mary's Hospital information technology department. These Western Eye Hospital notes were reviewed to ascertain whether any of the patients would have been on regular ocular medication at the time of their admission. The St Mary's Hospital notes of those that would have been on drops were checked in order to see whether these had been prescribed.

\section{Results}

One hundred and sixty six patients admitted to the medical or surgical units at St Mary's Hospital were also registered in the ophthalmology clinics at the Western Eye Hospital. Of these, 22 patients would have been expected to receive ocular medication during their admission. On the day of admission, only seven of 22 patients received the correct drops. Of the remaining 15, one patient was prescribed the correct drops two days after admission. One patient was only prescribed two out of the three drops he was receiving for glaucoma, and one was given betamethasone drops as Betnesol instead of as Betnesol-N (a preparation which includes neomycin). The other patients were not prescribed any drops. Excluding the patient who later received his drops, 14 out of 22 patients did not receive their correct ocular medication during their admission. These patients were on drops for the following reasons: glaucoma (5), post-cataract surgery (3), corneal exposure (3), bullous keratopathy (1), traumatic uveitis (1), and a recent conjunctival bleb leak after glaucoma surgery (1).

Review of the case notes also revealed that four patients had medical admissions that may have been related to their ocular medications. Each of these patients had been prescribed a topical $\beta$-blocker for glaucoma, but was admitted with a medical condition, which may have been aggravated by $\beta$-blockers. Three of the patients were admitted with heart failure while on levobunolol. In only one case, was there a documented discussion with an ophthalmologist regarding the use of an alternative drop. One of the other three patients did not receive the drops while an inpatient. Ophthalmology notes suggest that the drops were subsequently recommenced following discharge from the ward. The third patient continued his levobunolol while an inpatient. One other patient was admitted with a collapse of unknown origin, while on levobunolol. He did not, however, receive his eye drops while an inpatient. Two further patients, while receiving treatment for reversible airways disease, had been prescribed topical $\beta$-blockers, although in neither case was a cardiac or respiratory problem the reason for admission. Only one of these patients received their drops as an inpatient.

Therefore, up to six patients had been prescribed topical $\beta$-blockers inappropriately. However, in only one of these cases did the ophthalmic outpatient notes mention that the drops might have contributed to the medical problems or hospital admissions.

\section{Discussion}

Our results confirm that the majority of patients on eye drop therapy do not have their 
medications correctly prescribed during nonophthalmic admissions. Our initial aim was to assess the degree of correct prescribing, but we also found that six out of the 22 patients were receiving ocular medications inappropriately. There also appeared to be a failure of communication between the various doctors involved in the patients' management. These findings demonstrate the difficulties of disseminating relevant clinical information between doctors especially where the treatments are instituted at different hospitals or by different teams of doctors (including general practitioners). This problem should be reduced with the introduction of computerised records. However, at present, patients could be given a brief summary of their medical conditions and medications to keep, and produce at any consultation. Furthermore, doctors need to remember that before prescribing a new treatment they need to take a full drug history, which includes tablets, inhalers, and eye drops.

\section{Summary points}

- The majority of patients who have been prescribed eye drops do not receive them during non-ophthalmic admissions to hospital.

- A drug history should ascertain which tablets, inhalers, and eye drops a patient uses, as topical $\beta$-blockers continue to be inappropriately prescribed.

We would like to thank Lance McCarthy (Hospital Manager, Western Eye Hospital), St Mary's Hospital information technology department, and the medical records departments at St Mary's Hospital and the Western Eye Hospital.

1 Diggory P, Cassels-Brown A, Vail A, et al. Avoiding unsuspected respiratory side-effects of topical timolol with unsuspected respiratory side-effects of topical timolol with
cardioselective or sympathomimetic agents. Lancet cardioselective or

2 Price NC. Importance of asking about glaucoma. BMf 1983;286:349.

\section{Medical Anniversary}

Robert Koch, 5 October 1882

On this day, Robert Koch (1843-1910) isolated the tubercle bacillus, and deservedly won the Nobel Prize in 1905. He was born in 1843 in Klausthal, Prussia, studied medicine in Gottingen, and served as a field surgeon in the Franco-Prussian war. He came into prominence with the discovery of the bacterial cause of anthrax (1876) but the obsession which goaded him onwards was to unfold the cause of tuberculosis. On this very day, in the presence of two assistants, Loeffler and Gaffky, the surface of the serum jelly in the test tube vials, inoculated with tuberculous tissue, revealed tiny flecks. Microscopic examination revealed the crooked rods of tubercle bacilli and guinea pig inoculation confirmed the disease.-D G fames 